\title{
Adolescentes socialmente menos favorecidos estão mais sujeitos ao sedentarismo? uma revisão sistemática
}

\author{
Are socially disadvantaged teenagers more subject to sedentary lifestyles? a systematic review \\ ¿Los adolescentes socialmente desfavorecidos están más sujetos a estilos de vida sedentarios? una \\ revisión sistemática
}

Recebido: 20/10/2021 | Revisado: 28/10/2021 | Aceito: 01/11/2021 | Publicado: 02/11/2021

Fabiângelo de Moura Carlos ORCID: https://orcid.org/0000-0003-1392-2875 Universidade Federal de São João Del Rei, Brasil E-mail: fabiangelomc@gmail.com

Juscelino de Souza Borges Neto ORCID: https://orcid.org/0000-0002-4551-3278 Universidade Federal de São João Del Rei, Brasil E-mail: celinoborges1980@gmail.com

Juliana Mara Flores Bicalho ORCID: https://orcid.org/0000-0003-1445-8234 Universidade Federal de São João Del Rei, Brasil E-mail: jmfbicalho@gmail.com

Gustavo Henrique Oliveira ORCID: https://orcid.org/0000-0003-2779-2549 Centro Universitário Una, Brasil E-mail: contato.gholiveira@gmail.com

Cezenário Gonçalves Campos ORCID: https://orcid.org/0000-0001-5650-0096 Universidade Federal de São João Del Rei, Brasil E-mail: cezenario@yahoo.com.br

Wendell Costa Bila

ORCID: https://orcid.org/0000-0003-3048-5953 Universidade Federal de São João Del Rei, Brasil E-mail: wendellbil1@gmail.com Joel Alves Lamounier

ORCID: https://orcid.org/0000-0002-0581-3217 Universidade Federal de São João Del Rei, Brasil E-mail: lamounierjoel@gmail.com

Márcia Christina Caetano Romano ORCID: https://orcid.org/0000-0002-1819-4689 Universidade Federal de São João Del Rei, Brasil E-mail: marciachristinacs@gmail.com

\begin{abstract}
Resumo
O objetivo deste estudo foi identificar e sintetizar na literatura evidências da associação entre nível socioeconômico e nível de atividade física entre adolescentes. Trata-se de um estudo de revisão sistemática, realizado nas bases de dados Medline-Pubmed, Scopus e Lilacs. Utilizando os descritores e palavras-chaves adolescente, classe social, atividade física, no idioma Português e sua equivalência na língua Inglesa. A pergunta de pesquisa baseou-se no acrônimo PECO, que estabelece como Pessoas os adolescentes de 10 a 19 anos, a Exposição o nível socioeconômico, a Comparação os níveis socioeconômicos baixo/alto e os Outcomes (resultados) os níveis de atividade física. O processo de identificação, triagem, elegibilidade e inclusão dos artigos encontrados atendeu ao protocolo PRISMA. Foram identificados 2.243 artigos. Após aplicação dos critérios de inclusão e exclusão foram selecionados sete artigos, sendo todos internacionais e de delineamento transversal. A maior parte dos artigos evidenciou que adolescentes com menor nível socioeconômico estão mais sujeitos a serem sedentários. No entanto, não há um consenso sobre essa associação. Assim, são necessárias novas pesquisas para compreender essa relação com o intuito de favorecer e fomentar práticas de atividade física aos adolescentes no empenho contra o sedentarismo.
\end{abstract}

Palavras-chave: Adolescente; Classe social; Atividade física.

\section{Abstract}

The aim of this study was to identify and synthesize in the literature evidence of the association between socioeconomic level and level of physical activity among adolescents. This is a systematic review study, carried out in the MedlinePubmed, Scopus and Lilacs databases. Using descriptors and keywords adolescent, social class, physical activity, in the 
Portuguese language and its equivalence in the English language. The research question was based on the acronym PECO, which establishes as People the adolescents from 10 to 19 years old, the Exposure the socioeconomic level, the Comparison the low / high socioeconomic levels and the Outcomes (results) the levels of physical activity. The identification, screening, eligibility and inclusion process of the articles found complied with the PRISMA protocol. 2,243 articles were identified. After applying the inclusion and exclusion criteria, seven articles were selected, all of them international and cross-sectional. Most of the articles showed that adolescents with lower socioeconomic status are more likely to be sedentary. However, there is no consensus on this association. Thus, further research is needed to understand this relationship in order to favor and promote physical activity practices for adolescents in their efforts against sedentary lifestyle.

Keywords: Adolescent; Social class; Exercise.

\section{Resumen}

El objetivo de este estudio fue identificar y sintetizar evidencia en la literatura de la asociación entre nivel socioeconómico y nivel de actividad física en adolescentes. Se trata de un estudio de revisión sistemática realizado en las bases de datos Medline-Pubmed, Scopus y Lilacs. Utilizando los descriptores y palabras clave adolescente, clase social, actividad física, en lengua portuguesa y su equivalencia en lengua inglesa. La pregunta de investigación se basó en el acrónimo PECO, que define como Personas a los adolescentes de 10 a 19 años, Exposición el nivel socioeconómico, la Comparación los niveles socioeconómicos bajo / alto y los Resultados (resultados) los niveles de actividad física. El proceso de identificación, cribado, elegibilidad e inclusión de los artículos encontrados cumplió con el protocolo PRISMA. Se identificaron 2.243 artículos. Después de aplicar los criterios de inclusión y exclusión, se seleccionaron siete artículos, todos ellos internacionales y con un diseño transversal. La mayoría de los artículos mostraron que los adolescentes con un nivel socioeconómico más bajo tienen más probabilidades de ser sedentarios. Sin embargo, no hay consenso sobre esta asociación. Por lo tanto, se necesitan más investigaciones para comprender esta relación con el fin de favorecer y fomentar las prácticas de actividad física de los adolescentes en su lucha contra los estilos de vida sedentarios.

Palavras clave: Adolescente; Clase social; Ejercicio físico.

\section{Introdução}

Atividade física (AF) é qualquer movimento corporal produzido pelos músculos esqueléticos que requer gasto de energia, incluindo atividades realizadas durante o trabalho, brincadeiras, tarefas domésticas, envolvimento em atividades recreativas (World Health Organization [WHO], 2020). Neste aspecto, o termo AF não deve ser confundido com exercício físico, que consiste em uma subcategoria de AF planejada, estruturada, repetitiva e visa melhorar ou manter um ou mais componentes da aptidão física (Budde et al., 2016; WHO, 2020).

Níveis regulares e adequados de AF levam à melhora da aptidão muscular e cardiorrespiratória, favorecendo também a saúde óssea e funcional, reduzindo o risco de hipertensão arterial sistêmica, doença cardíaca coronariana, acidente vascular cerebral, diabetes, alguns tipos de câncer e a depressão. Além disso, reduz o risco de quedas, fraturas de coluna e quadril e são fundamentais para o equilíbrio energético e controle do peso corporal (WHO, 2020).

A prática de AF pode influenciar o desenvolvimento biológico, psicológico e social de crianças e adolescentes, possibilitando uma melhoria na saúde e consequentemente na qualidade de vida (Nagorny et al., 2018).

Apesar de serem inúmeros os benefícios da prática regular de AF para crianças e adolescentes, o sedentarismo vem aumentando em todo o mundo, representando grandes desafios para as autoridades em saúde pública (Tremblay et al., 2016). Um estudo realizado em 32 países, descrevendo os níveis de AF dos adolescentes, concluiu que a maioria dos púberes não atendia às recomendações de $\mathrm{AF}$ preconizadas para essa faixa etária (Kalman et al., 2015). Esse déficit nas práticas físicas gera a exposição a inúmeros prejuízos à saúde do adolescente, como depressão, doenças crônicas não transmissíveis (DCNT), eventos cardiovasculares, hipertensão arterial sistêmica (HAS), doença cardíaca coronariana, acidente vascular cerebral, diabetes mellitus (DM). Essa situação gera o aumento na demanda e gastos no serviço público de saúde (WHO, 2020).

No Brasil, esse grave problema também é evidenciado. A Pesquisa Nacional de Saúde (PNS) realizada no país em 2015 , em parceria com o Instituto Brasileiro de Geografia e Estatística (IBGE), concluiu que mais da metade dos jovens acima de 18 
anos estão com sobrepeso em consequência de alimentação inadequada e sedentarismo. Destaca-se também que o sobrepeso contribuiu com mais de 70\% das mortes no ano de 2012 (Instituto Brasileiro de Geografia e Estatística [IBGE], 2015).

Dentre os fatores que influenciam o volume de AF, o nível socioeconômico (NSE) vem sendo especialmente destacado, pois exerce influência nas atitudes pessoais, experiências vivenciadas, bem como na exposição a vários fatores de risco à saúde (Menezes \& Duarte, 2015). Nesse contexto, a presente investigação apresenta como objetivo identificar e sintetizar evidências da associação entre os NSE e de AF entre adolescentes.

\section{Material e Métodos}

Trata-se de uma revisão sistemática da literatura realizada no período de 01 a 09 de dezembro de 2020. Definiu-se a questão de pesquisa por meio da estratégia PECO e busca de evidência, que estabelece como "Pessoas" os adolescentes de 10 a 19 anos, a "Exposição" o nível socioeconômico, a "Comparação" os níveis socioeconômicos baixo/alto, os "Outcomes" (resultados) os níveis de atividade física. O processo de identificação, triagem, elegibilidade e inclusão dos artigos encontrados atendeu ao protocolo PRISMA - Preferred Reporting Items for Systematic Review and Meta-Analysis (Galvão et al., 2015; Ministério da Saúde, 2014; Moher et al., 2009).

As variáveis coletadas em cada artigo incluem o ano/país de publicação, o número de adolescentes em cada estudo, faixa etária específica, o tipo do estudo, instrumentos utilizados, conclusão e a existência de associação entre as variáveis de interesse. O nível de evidência científica dos estudos foi classificado conforme a categorização da Agency for Health Care Research and Quality (AHRQ; Stillwell et al., 2010).

Realizou-se a pesquisa nas bases de dados eletrônicas Medical Literature Analysis and Retrieval System Online (Medline), US National Library of Medicine National Institutes of Health (Pubmed), SciVerse Scopus (Scopus) e Literatura Latino-Americana e do Caribe em Ciências da Saúde (Lilacs), mesclando os termos integrantes da lista de Descritores em Ciências da Saúde (DeCs) e da Medical Subject Headings (MeSH), em português, "adolescente", "classe social", "atividade física/exercício" e em inglês, "adolescent", "social class", "physical activity/exercise", utilizando os operadores booleanos or e and.

A estratégia de busca no portal Pubmed/Medline incluiu: "adolescent" and "social class" and "physical activity" or "exercise", além de "social" and "physical activity"or "exercise". Nas bases de dados dos periódicos Scopus e Lilacs foram utilizados os termos "adolescent" and "social class" and "physical activity" or "exercise".

Foram definidos como critérios de inclusão para essa revisão os estudos nos idiomas inglês, espanhol e português que continham adolescentes como participantes da pesquisa, todos quantitativos, que associaram a relação entre NSE e AF. Foram também utilizados como critérios de exclusão relatos de caso de experiência. Não foi definido limite temporal para essa pesquisa. A busca foi realizada de maneira independente por dois pesquisadores.

\section{Resultados}

A pesquisa inicial nas bases de dados obteve 2.243 artigos, conforme apresentado na Figura 1, dentre os quais cinco foram excluídos por estarem em duplicata, restando 2.238 artigos. Após leitura de títulos e resumos, restaram para a leitura integral 22 artigos que atendiam os critérios de inclusão e destes 15 foram excluídos por não responderem à pergunta da pesquisa ou não atenderem aos critérios de inclusão na leitura integral. Portanto, foram incluídos nesta investigação sete artigos. 
Figura 1 - Fluxograma utilizado no processo de seleção dos artigos baseado no modelo PRISMA.

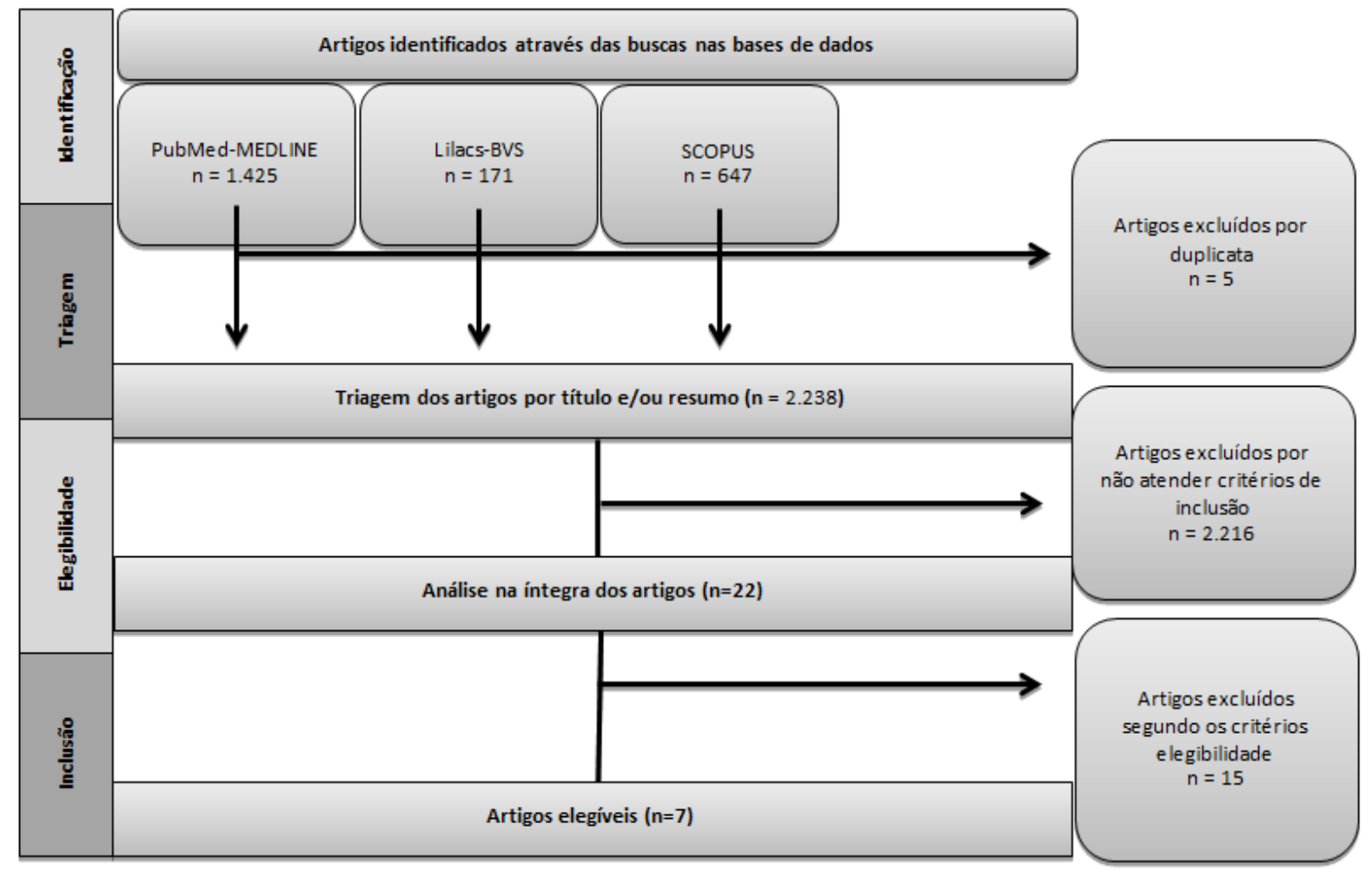

Fonte: Fluxograma baseado no guideline PRISMA.

Todos os sete artigos que compõem a presente investigação são de delineamento transversal, com nível de evidência três e com amostras internacionais. Diferentes instrumentos e parâmetros foram utilizados para mensurar o NSE e a AF, demonstrando, assim, grande heterogeneidade das variáveis nas análises de associação estudadas, conforme evidenciado no Quadro 1. 
Quadro 1 - Artigos incluídos nesta revisão conforme autoria e ano de publicação, país de origem, faixa etária dos participantes, instrumento utilizado para avaliar o desfecho e a associação entre as variáveis de interesse.

\begin{tabular}{|c|c|c|c|c|}
\hline $\begin{array}{l}\text { Autor e ano de } \\
\text { publicação }\end{array}$ & País - Região & $\begin{array}{l}\text { Faixa } \\
\text { etária } \\
(\text { anos })\end{array}$ & $\begin{array}{l}\text { Instrumento utilizado } \\
\text { para avaliar o } \\
\text { desfecho }\end{array}$ & Principais resultados \\
\hline KRIST et al., 2017 & Alemanha & $12-13$ & $\begin{array}{l}\text { HBSC (Health } \\
\text { Behaviour in School- } \\
\text { aged Children). }\end{array}$ & $\begin{array}{l}\text { Adolescentes desfavorecidos socialmente e } \\
\text { que residem em bairros cuja população } \\
\text { apresenta menor NSE tiveram maior } \\
\text { comportamento sedentário verificado através } \\
\text { do maior tempo de uso de tela. }\end{array}$ \\
\hline HANKONEN et al., 2017 & Finlândia & $16-19$ & Sistema COM-B & $\begin{array}{l}\text { Maior NSE relacionou-se a maior AF, sendo } \\
\text { fatores determinantes a oportunidade, acesso e } \\
\text { motivação para AF. }\end{array}$ \\
\hline EIME et al., 2015 & Austrália & 15 ou mais & $\begin{array}{l}\text { ERASS (Exercise, } \\
\text { Recreation and Sport } \\
\text { Survey) }\end{array}$ & $\begin{array}{l}\text { Menor NSE está associado com maior nível de } \\
\text { AF. As taxas de participação irregular em AF } \\
\text { aumentaram à medida que o NSE aumentava. }\end{array}$ \\
\hline COCKER et al., 2012 & Europa & $12-18$ & IPAQ (versão longa) & $\begin{array}{l}\text { Menor NSE está associado com menor nível } \\
\text { de AF, sendo que a baixa situação econômica } \\
\text { gera crenças e atitudes no adolescente que o } \\
\text { impedem de praticar mais AF. }\end{array}$ \\
\hline LÄMMLE et al., 2012 & Alemanha & $\begin{array}{c}6-9 \\
10-17\end{array}$ & $\begin{array}{l}\text { MoMotest (Motorik - } \\
\text { Module) }\end{array}$ & $\begin{array}{l}\text { Crianças e adolescentes com baixo NSE } \\
\text { apresentaram menor taxa de AF. }\end{array}$ \\
\hline VESELSKA et al., 2011 & Slovakia & $11-17$ & $\begin{array}{l}\text { HBSC } \\
\text { Behaviour in School- } \\
\text { aged Children) }\end{array}$ & $\begin{array}{l}\text { Adolescentes com alto NSE tiveram maior } \\
\text { nível de AF. }\end{array}$ \\
\hline $\begin{array}{l}\text { BORRACCINO et al., } \\
2009\end{array}$ & $\begin{array}{l}\text { Europa } \\
\text { América do Norte }\end{array}$ & 11,13 e 15 & $\begin{array}{l}\text { HBSC } \\
\text { Behaviour in School- } \\
\text { aged Children) }\end{array}$ & $\begin{array}{l}\text { Associação positiva, baixo NSE e baixa AF, } \\
\text { neste estudo que envolveu adolescentes de } 32 \\
\text { países os autores também constataram que as } \\
\text { meninas eram significativamente menos ativas } \\
\text { que os meninos e as crianças mais velhas eram } \\
\text { menos ativas quando comparadas às mais } \\
\text { novas. }\end{array}$ \\
\hline
\end{tabular}

Fonte: Autores.

Os estudos selecionados (Borraccino et al., 2009; Cocker et al., 2012; Eime et al., 2015; Hankonen et al., 2017; Krist et al., 2017; Lämmle et al., 2012; Veselska et al., 2011) perfizeram um total de 175.279 mil adolescentes e contemplaram todo o período da adolescência, incluindo dos 10 aos 19 anos de idade. Sobre a associação investigada, em 71,4\% dos artigos (Borraccino et al., 2009; Cocker et al., 2012; Hankonen et al., 2017; Lämmle et al., 2012; Veselska et al., 2011) evidenciou-se uma associação direta entre NSE e AF, dentre os quais 60\% dos estudos (Borraccino et al., 2009; Cocker et al., 2012; Lämmle et al., 2012) demonstraram que adolescentes com um baixo NSE estavam mais vulneráveis a uma maior inatividade e 40\% das investigações (Hankonen et al., 2017; Veselska et al., 2011) apontaram que os participantes com maior NSE se apresentavam mais ativos fisicamente.

É importante salientar que apenas um estudo mostrou que a prática de AF foi maior em adolescentes com menor NSE (Eime et al., 2015) e que um artigo não evidenciou associação entre essas variáveis (Krist et al., 2017).

Foram encontrados três instrumentos diferentes para mensurar o NSE, quais sejam o FAS (Family Affluence Scale), o IRSAD (Relative Socio-economic Advantage and Disadvantage) e o nível de escolaridade dos pais. O FAS foi o mais utilizado, totalizando $43 \%$ dos artigos selecionados. No que se refere à avaliação do nível de AF, foram utilizados cinco diferentes critérios, ou seja, o ERASS (Exercise Recreation and Sport Survey), o Motorik (Module MoMotest), o IPAQ (Questionário Internacional de Atividade Física, na sua versão longa), o HBSC (Health Behaviour in School-aged Children) e Sistema COM-B. Em 43\% dos artigos, destacou-se o questionário HBSC entre os instrumentos que avaliam a AF. 


\section{Discussão}

Os achados desta revisão apontam que adolescentes com um baixo NSE estão mais vulneráveis à inatividade física. Corroborando com este resultado, um estudo de Silva e colaboradores, pontuou que adolescentes de classe média têm menos chances de serem inativos quando comparados aos de classe baixa (Silva et al., 2018). Uma possível explicação para essa associação é que adolescentes frequentemente têm interesse por esportes e atividades físicas que apresentam um custo financeiro. Nesse caso, existe, muitas vezes, a necessidade de aquisição de equipamentos e pagamentos de mensalidades, além de custos com deslocamento. Assim, o acesso a tais atividades relaciona-se ao maior poder aquisitivo da família (Eime et al., 2015).

Aspecto também relevante relacionado à questão socioeconômica e os níveis de AF é a escolaridade dos pais, na medida em que famílias com maior NSE, cujos pais têm maior formação acadêmica e conhecimento sobre a saúde, estimulam mais os adolescentes a praticarem hábitos saudáveis, como a prática de $\mathrm{AF}$ (Christofaro et al 2018). O apoio e incentivo para esta prática é uma forma de assistência que auxilia na manutenção e/ou adoção desse comportamento, podendo ser ofertado de diferentes formas, como nos campos emocional, instrucional e instrumental. Pais com menor nível de escolaridade podem apresentar menor preocupação e atenção com a prática de AF de seus filhos, devido ao menor nível de conhecimento sobre a sua importância para a saúde, além da menor condição econômica para disponibilizar equipamentos, materiais e oportunidades para os seus filhos, assim como a elevada carga de trabalho semanal destes pais (Cabral et al., 2020).

Uma justificativa que talvez possa contribuir para o entendimento da relação entre baixo NSE e baixo nível de AF justamente é o contexto familiar. Uma investigação realizada no ano de 2016 mostrou que adolescentes, cujos pais praticavam esportes na infância e adolescência, estariam mais propensos a serem fisicamente ativos. A prevalência de adolescentes ativos no referido estudo foi maior quando ambos os pais estavam atualmente ativos e/ou teriam sido ativos no passado. A associação então entre a prática de AF pelos pais e adolescentes tem sido explicada pela reprodução por parte dos filhos, do comportamento, estímulo e apoio dos pais à prática de AF. Esse estímulo inclui apoio financeiro para fornecimento de equipamento esportivo ou pagamento de aulas de esportes, motivação verbal e provisão de transporte, facilitando o deslocamento (Dumith et al., 2016).

Outro ponto a ser destacado é que, em regiões menos favorecidas economicamente, a violência impede as atividades ao ar livre. O medo da violência favorece escolhas de atividades de baixo gasto calórico (Silva et al., 2018). De acordo com o Programa das Nações Unidas para o Desenvolvimento (PNUD), no Brasil, 24,7\% das pessoas já sofreram por restrição a locais de lazer devido ao medo de delinquência, provocando um impacto negativo na prática da AF de adolescentes e adultos (Programa das Nações Unidas para o Desenvolvimento Humano Nacional, 2017). Sendo assim, as elevadas taxas de violência no Brasil podem estar favorecendo escolhas de atividades sedentárias, como assistir televisão, jogar videogames e utilizar computadores (Cerqueira et al., 2017).

Para combater esse cenário, políticas públicas adequadas são de extrema importância, com opções urbanas e seguras de lazer ao ar livre, presença de educadores físicos e diversidade de opções de esportes e atividades, favorecendo o interesse e motivação do adolescente no sentido da permanência nessas atividades. A exposição a ambientes favoráveis pode contribuir com a redução do sedentarismo na adolescência, sendo então necessários investimentos públicos com um foco na melhoria do ambiente da vizinhança e da cidade (Schaefer et al., 2018).

Reforçando a questão de espaços adequados para AF, um estudo realizado com adolescentes na cidade de Curitiba, Paraná, no ano de 2015, demonstrou que a percepção sobre a existência de lugares para caminhar e andar de bicicleta tinha uma relação inversa com sedentarismo. Adolescentes de bairros com maior facilidade de deslocamento por bicicletas possuíam maior tempo despendido em atividades físicas (Smith et al., 2015).

Desta forma, intervenções para reduzir o sedentarismo entre adolescentes devem considerar o ambiente ao redor das suas casas, melhorando a quantidade e a qualidade das calçadas, ciclovias, parques e áreas que permitam caminhar ou andar de bicicleta. A exposição a ambientes favoráveis pode reduzir o sedentarismo nesta fase (Sarabia et al., 2018). Além disso, tais 
intervenções podem potencializar o transporte ativo para escola, o que certamente relaciona-se com índices mais elevados de prática de exercícios físicos na juventude (Gubelmann et al., 2018).

É importante ressaltar que as políticas públicas atuais para promoção da saúde não são efetivadas em sua integralidade, e especialmente não envolvem propostas mais amplas que poderiam contemplar o combate ao sedentarismo, na medida em que a infraestrutura adequada para o deslocamento ativo pode ser um fator importante para elevar os padrões de AF em adolescentes (Sarabia et al., 2018). Remodelar os ambientes para torná-los mais adequados à mobilidade dos adolescentes pode fazer parte de uma política ampla e de longo prazo destinada a melhorar o uso e o compartilhamento das ruas de forma sustentável e saudável (Sá et al., 2015).

Adicionalmente, pode-se destacar que adolescentes mais vulneráveis possuem outras demandas que ocupam o seu tempo, como o próprio trabalho. A inserção de crianças e adolescentes no mercado de trabalho é uma questão mundial, que ocorre em maior proporção nos países mais pobres e de renda média, como o Brasil. Em 2012, cerca de 265 milhões de crianças e adolescentes de cinco a 17 anos de idade eram economicamente ativos, com quase 168 milhões realizando trabalho em desacordo com a idade mínima estabelecida e 85 milhões exercendo algum trabalho considerado perigoso ou com potenciais efeitos nocivos para a saúde. Essa situação certamente traz dificuldades para que o adolescente consiga conciliar atividades profissionais e escolares, com a prática regular de AF (Piola et al., 2020).

É relevante também abordar o espaço da escola enquanto ambiente oportuno para a realização de AF pelos adolescentes, uma vez que estes permanecem grande parte do tempo naquele local, sendo, portanto, apropriado para o incentivo de uma vida ativa. De fato, a escola é um espaço institucional de convivência social que necessita acolher o escolar, com grande potencial para influenciar a adoção de modos de vida mais saudáveis. Portanto, investimentos devem ser feitos para a melhoria da infraestrutura física, material e qualificação de docentes, para potencializar esta capacidade da escola, enquanto veículo de promoção da saúde de adolescentes (Bastos et al., 2021; Mendonça et al., 2018).

Com foco na educação física e saúde, cabe à escola promover práticas e ações que visem incentivar a busca por uma vida saudável e prevenção do sedentarismo, chamando a atenção para a importância desta disciplina escolar. Acredita-se que, em muitos cenários, a Educação Física escolar apresenta-se como a única oportunidade de o aluno ter contato com as práticas corporais e se beneficiar das vantagens da orientação de um profissional. Desta forma, ela se torna um estímulo à promoção do esporte, jogos lúdicos, cultura corporal do movimento e qualquer atividade que promova a saúde integral dos estudantes (Oliveira et al., 2016).

Neste cenário, a educação física precisa ser cada vez mais valorizada, pois contribui significativamente para o desenvolvimento motor e cognitivo, além de influenciar positivamente na busca por hábitos mais saudáveis entre crianças e jovens, sendo considerada uma agente de socialização no estabelecimento de hábitos saudáveis (Calvo-Ortega \& Perrino-Peña, 2017). Portanto, é importante a presença de uma educação física diária e de qualidade (Nahas et al., 2016).

No presente estudo, foi também identificadas distintas formas de avaliação do nível de AF, predominantemente por meio de questionários validados para as avaliações, e cuja utilização é um ponto positivo nas investigações, pois contribui para a manutenção das propriedades psicométricas destes instrumentos, potencializando o rigor metodológico e as evidências encontradas (Fortes \& Araújo, 2019).

Vale destacar que, considerando-se os benefícios para a saúde de um estilo de vida fisicamente ativo durante a adolescência, a exemplo da melhoria da aptidão cardiorrespiratória, muscular, óssea e metabólica, o auxílio na perda de peso, melhoria do desenvolvimento cognitivo e comportamento pró-social, os hábitos nessa fase são transferidos para a idade adulta. Sendo assim, torna-se importante compreender as variáveis que interferem negativa ou positivamente na prática de AF nesta faixa etária (Australian Government Department of Health, 2019; Department of Health and Human Services, 2018). 
Assim, adolescentes em condições de vida precárias estão mais susceptíveis à inatividade física, embora não seja consensual essa relação, pois há estudos que evidenciam resultados diferentes, apontando que adolescentes com maior NSE possuem inclusive altos índices de sedentarismo (Dumith et al., 2016; Lord et al., 2015; Rodrigues et al., 2017; Wang et al., 2016).

O presente estudo possui como limitações o número reduzido de estudos que o compôs, impedindo generalizações, apesar do considerado rigor metodológico adotado. Torna-se também necessária a realização de mais estudos com adolescentes brasileiros, apontando a relação do NSE e de AF, na medida em que os estudos que compõem a presente revisão são internacionais. Além disso, são importantes as investigações que analisam os fatores preditores associados ao sedentarismo entre os púberes, objetivando-se o combate e controle dessas determinantes, no sentido da promoção da saúde dos adolescentes (Audi et al., 2018; Gomes et al., 2016).

\section{Considerações Finais}

Dessa forma, conclui-se que os adolescentes com menor nível socioeconômico estão mais sujeitos a apresentarem menor nível de atividade física.

É pertinente o conhecimento dessa relação enquanto importante indicador para a busca de soluções que contribuam para a orientação das práticas e intervenções em saúde no público adolescente, principalmente no ambiente escolar que é um espaço importante para este processo, assim como aprimorar políticas públicas direcionadas à promoção da saúde das crianças e adolescentes.

\section{Referências}

Audi, C. A. F., Santiago, M. S., Andrade, M. G. G., \& Francisco, P. M. S. B. (2018). Common mental disorder among incarcerated women: a study on prevalence and associated factors. Ciênc. Saúde coletiva, 23 (11), 3587-3596. https://doi.org/10.1590/1413-812320182311.30372016

Australian Government Department of Health. (2019). Australian 24-hour movement guidelines for children (5-12 years) and young people (13-17 years): an integration of physical activity, sedentary behaviour, and sleep. Canberra: Australian Government Department of Health. https://www.health.gov.au/sites/default/files/documents/2021/03/australian-24-hour-movement-guidelines-for-children-5-to-12-years-and-young-people-13-to17-years-an-integration-of-physical-activity-sedentary-behaviour-and-sleep.pdf

Bastos, P. O., Moreira Junior, J. J., Norjosa, M. E. S., Vasconcelos, M. J. C., Queiroz, M. L., (2021). Atuação do enfermeiro brasileiro no ambiente escolar: Revisão narrativa. Research, Society and Development, 10 (9). http://doi.org/10.33448/rsd-v10i9.18089

Borraccino, A., Lemma, P., Iannotti, R. J., Zambon, A., Dalmasso, P., Lazzeri, G., Giacchi, M., \& Cavallo, F. (2009). Socio-economic effects on meeting PA guidelines: comparisons among 32 countries. MedSci Sports Exerc, 41 (4). https://doi.org/10.1249/MSS.0b013e3181917722

Budde, H., Schwarz, R., Velasques, B., Ribeiro, P., Holzweg, M., Machado, S., Brazaitis, M., Staack, F., \& Wegner, M. (2016). The need for differentiating between exercise, physical activity, and training. Autoimmun Rev, 15 (1), 110-111. https://doi.org/10.1016/j.autrev.2015.09.004

Cabral, T. G., Moura, I. R. D., \& Farias Júnior, J. C. (2020). O estado nutricional modera a associação entre apoio social, autoeficácia e tempo de atividade física dos adolescentes? Cien. Saude Colet. https://doi.org/10.1590/1413-81232021269.26552020

Calvo-Ortega, E., \& Perrino-Peña, M. (2017). Hábitos físico desportivos dos adolescentes de Castilha e León. Movimento, 23 (4), 1341-1352. https://doi.org/10.22456/1982-8918.71852

Cerqueira, D., Lima, R. S., Bueno, S., Valencia, L. I., Hanashiro, O., Machado, P. H. G., \& Lima, A. S. (2017). Atlas da Violência 2017. Rio de Janeiro: IPEA. https://www.ipea.gov.br/portal/images/170609_atlas_da_violencia_2017.pdf

Christofaro, D. G. D., Andersen, L. B., Andrade, S. M., Barros, M. V. G., Saraiva, B. T. C., Fernandes, R. A., \& Ritti-Dias, R. M. (2018). Adolescents physical activity is associated with previous and current physical activity practice by their parents. J. Pediatr, 94 (1), 48-55. https://doi.org/10.1016/j.jped.2017.01.007

Cocker, K., Artero, E. G., Henauw, S., Dietrich, S., Gottrand, F., Béghin, L., Hagströmer, M., Sjöström, M., Plada, M., Manios, Y., Mauro, B., Molnár, D., Moreno, L. A., Ottevaere, C., Valtueña, J., Maes, L. \& Bourdeaudhuij, I. (2012). Can differences in physical activity by socio-economic status in European adolescents be explained by differences in psychosocial correlates? A mediation analysis within the HELENA (Healthy Lifestyle in Europe by Nutrition in Adolescence) Study. Public Health Nutr, 15 (11), 2100-2109. https://doi.org/10.1017/s1368980012001036

Department of Health and Human Services. (2018). Physical Activity Guidelines Advisory Committee Scientific Report. Washington, DC: US. https://health.gov/sites/default/files/2019-09/PAG_Advisory_Committee_Report.pdf 
Dumith, S. C., Santos, M. N., Teixeira, L. O., Cazeiro, C. C., Mazza, S. E. I., \& Cesar, J. A. (2016). Prática de atividade física entre jovens em município do semiárido no Brasil. Ciênc. Saúde Colet, 21 (4), 1083-1093. https://doi.org/10.1590/1413-81232015214.18762015

Eime, R. M., Charity, M. J., Harvey, J. T., \& Payne, W. R. (2015). Participation in sport and physical activity: associations with socio-economic status and geographical remoteness. BMC Public Health. https://doi.org/10.1186/s12889-015-1796-0

Felden, E. P. G., Filipin, D., Barbosa, D. G., Andrade, R. D., Meyer, C., Beltrame, T. S., \& Pelegrini, A. (2016). Adolescentes com sonolência diurna excessiva passam mais tempo em comportamento sedentário. RevBras Med Esporte, 22 (3), 186-190. https://doi.org/10.1590/1517-869220162203147290

Fortes, C. P. D. D. \& Araújo, A. P. Q. C. (2019). Checklist para tradução e Adaptação Transcultural de questionários em saúde. Cad. saúde colet., 27 (2), 202209. https://doi.org/10.1590/1414-462X201900020002

Galvão, T. F., Pansani, T. S. A., \& Harrad, D. (2015). Principais itens para relatar Revisões sistemáticas e Meta-análises: A recomendação PRISMA. Epidemiol. Serv. Saúde, 24 (2), 335-342. http://doi.org/10.5123/S1679-49742015000200017

Gubelmann, C., Marques-Vidal, P., Bringolf-Isler, B., Suggs, L. S., Vollenweider, P., \& Kayser, B. (2018). Correlates of weekday compliance to physical activity recommendations in Swiss youth non-compliant in weekend days. Preventive Medicine Reports, 9, 86-91. https://doi.org/10.1016/j.pmedr.2017.12.004

Hankonen, N., Heino, M. T. J., Kujala, E., Hynynen, S., Absetz, P., Araújo-Soares, V., Borodulin, K., \& Haukkala, A. (2017). What explains the socioeconomic status gap in activity? Educational differences in determinants of physical activity and screentime. BMC Public Health 17:144. https://doi.org/10.1186/s12889016-3880-5

Instituto Brasileiro de Geografia e Estatística, Diretoria de Pesquisas, Coordenação de População e Indicadores Sociais. (2015). Pesquisa nacional de saúde do escolar 2015., IBGE. https://biblioteca.ibge.gov.br/visualizacao/livros/liv97870.pdf

Kalman, M., Inchley, J., Sigmundova, D., Iannotti, R. J., Tynjälä, J. A., Hamrik, Z., Haug, E., \& Bucksch, J. (2015). Secular trends in moderate-to-vigorous physical activity in 32 countries from 2002 to 2010: a cross-national perspective. Eur J Public Health, 25 (2), 37-40. https://doi.org/10.1093/eurpub/ckv024

Krist, L., Bürger, C., Ströbele-Benschop, N., Roll, S., Lotz, F., Rieckmann, N., Müller-Nordhorn, J., Willich, S. N., \& Müller-Riemenschneider, F. (2017). Association of individual and neighbourhood socioeconomic status with physical activity and screen time in seventh grade boys and girls in Berlin, Germany: a cross-sectional study. BMJ Open, 7. https://doi.org/10.1136/bmjopen-2017-017974

Lämmle, L., Worth, A., \& Bös, K. (2012). Socio-demographic correlates of physical activity and physical fitness in German children and adolescents. Eur. j. public health, 22 (6), 880-884. https://doi.org/10.1093/eurpub/ckr191

Lord, S., Manlhiot, C., Tyrrell P. N., Dobbin, S., Gibson, D., Chahal, N., Stearne, K., Fisher, A., \& McCrindle, B. W. (2015). Lower socioeconomic status, adiposity and negative health behaviours in youth: A cross-sectional observational study. BMJ Open, 5. https://doi.org/10.1136/bmjopen-2015-008291

Mendonça, G., Cheng, L. A., \& Farias Júnior, J. C. (2018). Padrões de prática de atividade física em adolescentes de um município da região Nordeste do Brasil. Ciênc. saúde coletiva, 23 (7), 2443-2451. https://doi.org/10.1590/1413-81232018237.21782016

Menezes, A. S., \& Duarte, M. F. S. (2015). Condições de vida, inatividade física e conduta sedentária de jovens nas áreas urbana e rural. RevBras Med Esporte, 21 (5), 338-344. https://doi.org/10.1590/1517-869220152105145322

Ministério da Saúde. (2014). Diretrizes Metodológicas: elaboração de revisão sistemática e metanálise de estudos observacionais comparativos sobre fatores de risco e prognóstico / Ministério da Saúde, Secretaria da Ciência, Tecnologia e Insumos Estratégicos, Departamento de Ciências e tecnologia - Brasília. https://bvsms.saude.gov.br/bvs/ct/PDF/diretrizes_metodologias_estudos_observacionais.pdf

Moher, D., Liberati, A., Tetzlaff, J., Altman, D. G., \& The PRISMA Group (2009). Preferred reporting items for systematic reviews and meta-Analyses: the PRISMA statement. PLoS Med, 21 (6). https://doi.org/10.1371/journal.pmed.1000097

Nagorny, G. A. K., Aguiar, P. S., Dias, A. F., Mello, J. B., Gaya, A. R., \& Gaya, A. C. A. (2018). Contribuição da Educação física escolar para o nível de atividade física diária. Revista Brasileira Prescrição Fisiologia do $\quad$ Exercício, $12 \quad 72$, $70-77$. http://www.rbpfex.com.br/index.php/rbpfex/article/view/1344/1022

Nahas, M., Silva, S., \& Garcia, M. (2016). Atividade física na infância e adolescência, Researchgate 11. 143-160. https://www.researchgate.net/publication/259922454_Atividade_fisica_na_infancia_e_adolescencia

Oliveira, L. F. L., \& Costa, C. R. B. (2016). Educação física escolar e a obesidade infantil. Rev. Científica Multidisciplinar Núcleo do Conhecimento, 10 (1), 87-101. https://www.nucleodoconhecimento.com.br/educacao/obesidade-infantil

Piola, T. S., Bacil, E. D. A., Pacífico, A. B., Camargo, E. M., \& Campos, W. (2020). Nível insuficiente de atividade física e elevado tempo de tela em adolescentes: impacto de fatores associados. Ciênc. saúde coletiva, 25 (7), 2803-2812. https://doi.org/10.1590/1413-81232020257.24852018

Programa das Nações Unidas para o Desenvolvimento. (2017). Relatório de Desenvolvimento Humano Nacional Movimento é Vida: Atividades Físicas e Esportivas para Todas as Pessoas: 2017. http://www.each.usp.br/gepaf/wp-content/uploads/2017/10/PNUD_RNDH_completo.pdf

Rodrigues, P. F., Melo, M., Assis, M., \& Palma, A. (2017). Condições socioeconômicas e prática de atividades físicas em adultos e idosos: uma revisão sistemática. Rev. Bras. Ativ. Fís. Saúde, 22 (3), 217-232. https://doi.org/10.12820/rbafs.v.22n3p217-232

Sá, T. H., Garcia, L. M. T., Mielke, G. I., Rabacow, F. M., \& Rezende, L. F. M. (2015). Changes in travel to school patterns among children and adolescents in the São Paulo Metropolitan Area, Brazil, 1997-2007. Journal of Transport \& Health, 2 (2), 143-150. https://doi.org/10.1016/j.jth.2015.02.008

Sarabia, T. T., Reis, M. S., Gonçalves, P. B., Reis, R. S. (2018). Tempo sedentário e ambiente percebido sobre o bairro em adolescentes de 12 a 17 anos. Rev. Bras. Cineantropom. Desempenho Hum., 20 (5), 456-467. https://doi.org/10.5007/1980-0037.2018v20n5p456 
Research, Society and Development, v. 10, n. 14, e305101422048, 2021

(CC BY 4.0) | ISSN 2525-3409 | DOI: http://dx.doi.org/10.33448/rsd-v10i14.22048

Schaefer, R., Barbiani, R., Nora, C. R. D., Viegas, K., Leal, S. M. C., Lora, P. S., Ciconet, R., \& Micheletti, V. D. (2018). Adolescent and youth health policies in the Portuguese-Brazilian context: specificities and approximations. Ciênc. Saúde Coletiva, 23 (9), 2849-2858. https://doi.org/10.1590/141381232018239.11202018

Silva, J., Andrade, A., Capistrano, R., Lisboa, T., Andrade, R. D., Felden, E. P. G., \& Beltrame, T. S. (2018). Níveis insuficientes de atividade física de adolescentes associados a fatores sociodemográficos, ambientais e escolares. Ciênc. saúde coletiva, 23 (12), 4277-4288. https://doi.org/10.1590/1413812320182312.30712016

Smith, N. R., Lewis, D. J., Fahy, A., Eldridge, S., Taylor, S. J. C., Moore, D. G., Clark, C., Stansfeld, S. A., \& Cummins, S. (2015). Individual socio-demographic factors and perceptions of the environment as determinants of inequalities in adolescent physical and psychological health: the Olympic Regeneration in East London (ORiEL) study. BMC Public Health, 15 (150). https://doi.org/10.1186/s12889-015-1459-1

Stillwell, S. B., Fineout-Overholt, E., Melnyk, B. M., \& Williamson, K.M. (2010). Evidence-Based Practice: Step by Step: The Seven Steps of Evidence-Based Practice. Am J Nurs, 110 (5). https://journals.lww.com/ajnonline/Fulltext/2010/01000/Evidence_Based_Practice_Step_by_Step__The_Seven.30.aspx

Tremblay, M. S., Barnes, J. D., González, S. A., Katzmarzyk, P. T., Onywera, V. O., Reilly, J. J., Tomkinson, G. R., \& The Global Matrix 2.0 Research Team. (2016). Global Matrix 2.0: report card grades on the physical activity of children and youth comparing 38 countries. J. Phys. Act. \& Health, 13 (2), $343-66$. https://doi.org/10.1123/jpah.2016-0594

Veselska, Z., Geckova, A. M., Reijneveld, S. A., \& van Dijk, J. P. (2011). Socio-economic status and physical activity among adolescents: the mediating role of self-esteem. Public Health, 125 (11), 763-8. https://doi.org/10.1016/j.puhe.2011.09.007

Wang, X., Hui, Z., Terry, P. D., Ma, M., Cheng, L., Deng, F., \& Zhang, B. (2016). Correlates of Insufficient Physical Activity among Junior High School Students: A Cross-Sectional Study in Xi'an, China. Int J Environ Res Public Health, 13 (4), 397. https://doi.org/10.3390/ijerph13040397

World Health Organization. Physical activity and sedentary behaviour. (2020). https://www.who.int/publications/i/item/9789240015128 